0.16 to 0.29 . Adverse events were reported mainly with use of opioids. They were cough, breath holding and chest tightness. The overall rate of complications was low. Both pharmacological and non-pharmacological methods were effective in reducing pain on injection of rocuronium. However, no one intervention can be considered superior over the other as the findings are not robust enough.

\section{Effect of intravenous dexmedetomidine in attenuation of hemodynamic response to intubation and skull-pin head holder application in patients undergoing elective neurosurgery}

\section{Amruta Ajgaonkar, Rajashree Gandhe}

Department of Anaesthesia, Kokilaben Dhirubhai Ambani Hospital and Medical Research Institute, Mumbai, Maharashtra, India

Background: The aim of this study is to determine the effect of intravenous dexmedetomidine in attenuation of hemodynamic response to intubation and skull-pin head holder application in patients undergoing elective neurosurgery. Materials and Methods: After approval from the hospital ethics committee, a prospective, randomized, double blinded study was conducted on 40 patients of ASA grades I or II, 18-65 years of age and undergoing elective neurosurgery. The intervention group received dexmedetomidine at $1 \mathrm{mcg} / \mathrm{kg} / \mathrm{hr}$ for 10 minutes followed by $0.5 \mathrm{mcg} / \mathrm{kg} / \mathrm{hr}$. The controls received normal saline at same rate. After standard induction, heart rate and mean arterial pressure were monitored at specified intervals during intubation and pin application. Intraoperative anesthetic and analgesic requirements were recorded. Complications, if any, were also noted. Appropriate statistical analysis was done. Data is presented as mean [SD]. Results: Demographics were comparable in both the groups. At one minute post-intubation, mean heart rate [hr] in control was 88.95 [15.05] and that in dexmedetomidine group 73.55 [11.70] $(P=0.001)$. The mean arterial pressure [MAP] in control was 105.35 [18.98] whereas that in dexmedetomidine group was 92.50 [4.38] $(P=0.020)$. On application of skull pin head holder, mean HR in control group was 93.2 [13.99] and 82.40 [11.18] in dexmedetomidine group $(P=0.01)$. MAP after application of skull pin head holder was 121.45 [SD 19.79] in control and 101.85 [SD 10.60] in dexmedetomidine group. Additional mean fentanyl requirement was $90 \mathrm{mcg}$ [49.74] in control group and $16 \mathrm{mcg}$ [34.70] in dexmedetomidine group $(P<0.00010)$. Conclusion: Intravenous dexmedetomidine is beneficial in attenuating the pressor response to intubation as well as application of skull pin head holder, without any serious adverse events. It also reduces the opioid and propofol requirement for the same.

\section{Comparison of oral intubation using flexible fibreoptic bronchoscope with or without rigid cervical collar: A clinical study}

Nitesh Gill, Shobha Purohit

Department of Anaesthesia, S M S Medical College, Jaipur, Rajasthan, India

Background: Device like fibreoptic bronchoscope is gold standard for difficult intubation situations. It can be performed by both orotracheal and nasotracheal route. But, through nasal route there are more chances of nasal bleeding and pressure necrosis while through oral route there is one problem which is clearing of upper airway for bronchoscope. Manoeuvres like jaw thrust and chin lift are very useful in clearing upper airway for bronchoscope. The aim of this study is to compare ease of oral intubation using flexible fibreoptic bronchoscope with or without rigid cervical collar, in terms of need of maneuvers: Jaw thrust and Chin lift. Materials and Methods: Twenty five patients in age range 20-50 years, of ASA I - $\Pi$, and of either sex undergoing elective surgery under general anesthesia were randomly allocated into each group. There were two groups: Group A (Rigid Cervical Collar) and group B (Without Collar). Intubating condition was assessed in between these groups with need of manoeuvres like jaw thrust and chin lift. Quantitative data is presented as mean values and standard deviation. Intergroup comparison of quantitative data was done by student's $t$-test and probability was considered to be significant if less than $<0.05$. Categorical data and need of manoeuvres (jaw thrust and chin lift) are presented as numbers and were compared among groups using Chi square test. $P<0.05$ was considered statistically significant. Results: There was

\begin{tabular}{lcccc}
\hline & $\begin{array}{c}\text { Group } \\
\text { A (with } \\
\text { collar) } \\
\boldsymbol{n = 2 5}\end{array}$ & $\begin{array}{c}\text { Group B } \\
\text { (without } \\
\text { collar) } \\
\boldsymbol{n = 2 5}\end{array}$ & Total & $\begin{array}{c}(\boldsymbol{P} \\
\text { value) }\end{array}$ \\
\hline $\begin{array}{l}\text { Need of } \\
\text { manoeuvres } \\
\text { (Jaw thrust and } \\
\text { chin lift) }\end{array}$ & 8 & 19 & 27 & 0.001 \\
$\begin{array}{l}\text { No need of } \\
\text { manoeuvres } \\
\begin{array}{l}\text { Jaw thrust and } \\
\text { chin lift) }\end{array}\end{array}$ & 17 & 6 & 23 & \\
Total & 25 & 25 & 50 & \\
\hline
\end{tabular}


significant difference found between group A and group B in terms of use of manoeuvres (jaw thrust and chin lift) for ease of intubation and clearing upper airway. In group B need of jaw thrust and chin lift for clearing airway is significantly higher than collar group.

\section{Intergroup comparison of need of manoeuvers} (Jaw thrust And Chin lift)

Conclusion: We concluded that as far as the oral intubation with flexible fibreoptic bronchoscope is concerned, rigid cervical collar is very useful tool for making intubation easier.

\section{A comparative study of hemodynamic responses to endotracheal extubation with prior treatment of lidocaine, verapamil and their combination}

Ambika Kumari, Rajeev Sharma

Department of Anaesthesia, S M S Medical College, Jaipur, Rajasthan, India

Background: Transient but significant undesirable hemodynamic effect such as hypertension and tachycardia persisting into the recovery period may be caused by tracheal extubation. Most patients tolerate these effects without any significant consequences, but some show an exaggerated response which is poorly tolerated and may lead to myocardial ischemia, cardiac de-compensation, pulmonary edema or cerebral hemorrhage. Lidocaine attenuates the hemodynamic response to tracheal extubation by its direct myocardial depressant effect, central stimulant effect, and peripheral vasodilatory effect and finally it suppresses the cough reflex. Verapamil controls hypertension and tachycardia by its direct vasodilatory and negative chronotropic. The aim of this study was to compare the blood pressure and heart rate responses to extubation with prior treatment of either lidocaine, verapamil or their combination. Materials and Methods: The study included 50 patients (age 20-50 years) in each four groups (A, B, C, D) undergoing elective surgery of the duration 1-2 hours. Group A was given verapamil $0.1 \mathrm{mg} / \mathrm{kg}$, group $B$ was given lidocaine $1 \mathrm{mg} / \mathrm{kg}$, group C was given $0.1 \mathrm{mg} / \mathrm{kg}$ verapamil plus $1 \mathrm{mg} / \mathrm{kg}$ lidocaine, group D was taken as control and was given normal saline. On completion of surgery, anesthetic agents were discontinued, residual muscle relaxation was reversed with neostigmine $0.05 \mathrm{mg} / \mathrm{kg}$ and atropine $0.02 \mathrm{mg} / \mathrm{kg} \mathrm{IV}$. Three minutes later, the study drug was given intravenously. Recovery from muscle relaxation was assessed by peripheral nerve stimulator. The trachea was then extubated two minutes after administration of the study drugs and $100 \%$ oxygen was given via face mask for five minutes.
Systolic and diastolic blood pressure and HR measured at the end of surgery, three minutes after the injection of neostigmine-atropine mixture, at the time of tracheal extubation, until 20 minutes after extubation were noted and compared among the four groups. The values at the time of tracheal extubation were also compared with baseline values within individual study groups. Results: In our study hemodynamic responses were blunted to tracheal extubation by verapamil alone and verapamil + lidocaine combination. However, there was no difference in between these two. But, in the lidocaine only group, there were significant increase in systolic blood pressure $(P<0.001)$ and heart rate $(P<0.001)$ at the time of tracheal extubation from the baseline values, and systolic blood pressure was also found significantly raised in lidocaine group in comparison to verapamil + lidocaine group. Conclusion: This study concludes that verapamil + lidocaine group combination or verapamil alone is better than lidocaine alone in attenuating the hemodynamic response to tracheal extubation.

\section{9. "Unveiling the puff of smoke": An observational study of anesthesia management of patients of moyamoya disease at a tertiary care centre}

\author{
Anita Shetty, Avinash Bajaj, Shashikant Shinde, \\ Shrikanta Oak, Madhu Garasia \\ Department of Anaesthesiology, Seth G.S. Medical College \\ and KEM Hospital, Parel, Mumbai, Maharashtra, India
}

Background: Moyamoya disease is a progressive bilateral stenosis and stroke and/or occlusion of the internal carotid artery or the terminal branches with formation of extensive collateral vessels at the base of the brain. Materials and Methods: An observational study of 12 pediatric patients with Moyamoya disease who underwent 18 Encephalodural Arteriosyngiosis (EDAS) procedure at our institute over a period of five years from 2008-2013. Results: Eighteen surgeries were performed on 12 patients. In three patients, bilateral EDAS was done in the same sitting. In six patients, EDAS was done bilaterally over a period of six months and in three patients, unilateral EDAS was done. One patient deteriorated neurologically in the postoperative period.

Conclusion: The likely contributory factors for neurological deterioration were history of preoperative infarction, bilateral EDAS in the same sitting, long duration of anesthesia and surgery, episodes of hypotension and decreased urine output. 